\title{
Factors encouraging mobile instant messaging service use in medical education
}

\author{
Kidong Kim ${ }^{1}$, Banghyun Lee ${ }^{\text {Corresp., }}{ }^{2}$, Youngmi Park ${ }^{3}$, Eun Young Jung ${ }^{4}$, Seul Ki Kim ${ }^{1}$, Dong Hoon Suh ${ }^{1}$, Bo Ram \\ Choi $^{2}$ \\ ${ }^{1}$ Department of Obstetrics and Gynecology, Seoul National University Bundang Hospital, Seongnam-Si, Gyeonggi-Do, South Korea \\ Department of Obstetrics and Gynecology, Hallym University, Kangdong Sacred Heart Hospital, Seoul, South Korea \\ 3 Division of Statistics, Medical Research Collaborating Center, Seoul National University Bundang Hospital, Seongnam-Si, Gyeonggi-Do, South Korea \\ 4 Win Women's Hospital, Suwon-Si, Gyeonggi-Do, South Korea \\ Corresponding Author: Banghyun Lee \\ Email address: banghyun.lee@gmail.com
}

Background. Mobile instant messaging services are being increasingly used for educational purposes, but their effectiveness in medical education is not well known. We assessed whether students' use of Kakao Talk (a mobile instant messaging service) during the early period of a week of clinical education influenced its use for academic purposes during a later period of the same week.

Methods. The online communication records of 151 third-year medical students (in 39 clinical education groups) who used Kakao Talk during clinical education were reviewed. The 39 groups were categorized as low, middle, or high according to the number of total chats (on all subjects, not just academic) per student over five days. The relationship between the number of total chats during the first two days and the number of academic chats during the last three days (of five-day chatroom weeks) was analyzed.

Results. The number of total and academic chats over all five days, the first two days, and the last three days was highest in groups with the highest number of total chats per student. Similarly, the highest number of students posting total and academic chats was found in these groups. In addition, the number of academic chats per student and the frequency of questions raised by students were also highest in these groups. During the last three days, the number of students posting total chats was lower than that during the first two days, and the number of academic chats per student posting academic chats was higher. The number of total chats on the first or second day positively correlated with the maximum value of academic chats on the third to fifth days.

Conclusion. High frequency mobile instant messaging use early on in clinical education might encourage its use for academic purposes during later periods. 
1 Factors encouraging mobile instant messaging service use in medical education

2

3 Kidong Kim ${ }^{1}$, Banghyun Lee ${ }^{2 *}$, Youngmi Park ${ }^{3}$, Eun Young Jung 4 , Seul Ki Kim ${ }^{1}$, Dong Hoon

$4 \mathrm{Suh}^{1}$, and Bo Ram Choi ${ }^{2}$

5

$6{ }^{1}$ Department of Obstetrics and Gynecology, Seoul National University Bundang Hospital, 82,

7 Gumi-ro 173 beon-gil, Bundang-gu, Seongnam-Si, Gyeonggi-Do, Republic of Korea

$8{ }^{2}$ Department of Obstetrics and Gynecology, Hallym University, Kangdong Sacred Heart

9 Hospital, 150, Seongan-ro, Gangdong-gu, Seoul, Republic of Korea

$10{ }^{3}$ Division of Statistics, Medical Research Collaborating Center, Seoul National University

11 Bundang Hospital, 82, Gumi-ro 173 beon-gil, Bundang-gu, Seongnam-Si, Gyeonggi-Do,

12 Republic of Korea

$13{ }^{4}$ Win Women's Hospital, 32, Bongyeong-ro 1759, Yeongtong-gu, Suwon-Si, Gyeonggi-Do,

14 Republic of Korea

Running title: Mobile instant messaging use in medical education

\section{* Corresponding author:}

19 Banghyun Lee, M.D., Ph.D.

Department of Obstetrics and Gynecology, Hallym University Kangdong Sacred Heart Hospital,

21 150, Seongan-ro, Gangdong-gu, Seoul, Republic of Korea

Tel.: 82-2-2152-1025 
23 Fax: 82-2-2224-2265

24 E-mail: banghyun.lee@gmail.com

25

26

27

28

29

30

31

32

33

34

35

36

37

38

39

40

41

42

43

44

45 Abstract

Peer) reviewing PDF | (2019:01:34282:1:2:NEW 4 Jun 2019) 
46 Background. Mobile instant messaging services are being increasingly used for educational

47 purposes, but their effectiveness in medical education is not well known. We assessed whether students' use of Kakao Talk (a mobile instant messaging service) during the early period of a

49 week of clinical education influenced its use for academic purposes during a later period of the 50 same week.

51 Methods. The online communication records of 151 third-year medical students (in 39 clinical 52 education groups) who used Kakao Talk during clinical education were reviewed. The 39 groups 53 were categorized as low, middle, or high according to the number of total chats (on all subjects, 54 not just academic) per student over five days.

55 The relationship between the number of total chats during the first two days and the number of 56 academic chats during the last three days (of five-day chatroom weeks) was analyzed.

57 Results. The number of total and academic chats over all five days, the first two days, and the

58 last three days was highest in groups with the highest number of total chats per student.

59 Similarly, the highest number of students posting total and academic chats was found in these 60 groups. In addition, the number of academic chats per student and the frequency of questions 61 raised by students were also highest in these groups. During the last three days, the number of 62 students posting total chats was lower than that during the first two days, and the number of 63 academic chats per student posting academic chats was higher. The number of total chats on the 64 first or second day positively correlated with the maximum value of academic chats on the third 65 to fifth days.

66 Conclusion. High frequency mobile instant messaging use early on in clinical education might 67 encourage its use for academic purposes during later periods. 


\section{Introduction}

91 Worldwide, the use of mobile instant messaging (MIM) services is rapidly increasing. Their 
92 relative convenience, accessibility, and capacity to disseminate a large amount of information in

93 various formats suggest that MIM services have high potential for educational use. Thus, the use

94 of this service in educational contexts seems inevitable. In many studies, the influence of MIM

95 use on students' academic performances has been shown to be positive, to be negative, or to have no effect, regardless of factors such as the level of students' education (Alipour et al., 2012;

Lauricella \& Kay, 2013; Bouhnik \& Deshen, 2014; Yeboah \& Ewur, 2014; Jamal et al., 2016;

So, 2016; Goyal, Tanveer, \& Sharma, 2017; Raiman, Antbring, \& Mahmood, 2017; Clavier et al., 2019). Several studies have reported that university students and faculties have positive perceptions of MIM services and accept their use in teaching and learning (Lauricella \& Kay, 2013; Bouhnik \& Deshen, 2014; Yeboah \& Ewur, 2014; So, 2016).

In the field of medical education, a few small studies have reported that MIM might be an effective way to acquire medical knowledge and a useful, feasible, and acceptable tool for medical students and residents (Alipour et al., 2012; Jamal et al., 2016; Goyal, Tanveer, \& Sharma, 2017; Raiman, Antbring, \& Mahmood, 2017). However, other studies showed MIM services to be ineffective tools in educating medical residents, suggesting instead that they served as a distraction (Clavier et al., 2019). Moreover, social media such as WhatsApp Messenger (a MIM service, WhatsApp Inc., Mountain View, California, USA), YouTube (YouTube, LLC., San Bruno, California, USA), and Twitter (Twitter, Inc., San Francisco, California, USA) showed no influence on the academic performance of medical students (AlFaris et al., 2018).

Given the growing use of MIM services in education, it is necessary to clarify their possible roles in medical education. utilized by about $93 \%$ of smartphone owners of all ages in South Korea (Nay, 2013). This free 
115 MIM service, which is available in 15 languages, includes free text messaging and free voice 116 calls (Han \& Cho, 2015; Unuth, 2019). One might expect that Kakao Talk would be useful in 117 medical education because of its ubiquity in South Korea for interpersonal and person-to-group 118 communication as well as for information sharing.

This study intended to determine the efficacy of the use of Kakao Talk by senior medical students during their clinical education. On the basis of its ubiquity, accessibility, and convenience, we assumed that Kakao Talk would be frequently used for academic purposes when it is seen as being helpful. We further hypothesized that an increase in student activity on Kakao Talk would be related to its later use for academic purposes. This is because it is likely that students who want to improve their clinical education would post more academically related chats once they become familiar with a learning environment that uses MIM.

\section{Materials and Methods}

\section{Sample}

This retrospective and exploratory pilot study used data on senior medical students in the clinical phase of their education at the Seoul National University Bundang Hospital. Data were collected between November 10, 2014 and December 5, 2015. The Institutional Review Board of Seoul National University Bundang Hospital approved the study design (No. L-2016-1265) on November 15, 2016. Informed consent was waived.

Participants were third-year medical students $(n=151)$ receiving clinical education in the Department of Obstetrics and Gynecology. Moreover, professors $(n=3$; two oncologists and one endocrinologist) and fellows $(n=2$; one oncologist and one obstetrician) majoring in obstetrics and gynecology as well as residents $(n=12)$ in that department also participated in the 
138 use of Kakao Talk for educational purposes.

\section{Study design}

140 Third-year medical students at this institution receive clinical education in the Department of

141 Obstetrics and Gynecology (a one-week rotation, Monday through Friday) in groups of three to

142 five students. Each student is assigned to an academic advisor who is a professor of obstetrics

143 and gynecology. The students attend in the delivery room, operating room, infertility

144 intervention room, wards, and outpatient clinic. On Thursdays, they attend an obstetrics and

145 gynecology course. Additionally, on Monday of the rotation week, each student is assigned the

146 medical case of a patient admitted to the obstetrics and gynecology ward. He or she presents that

147 case at a conference held on Friday of the same week (Figure 1).

148 We posited that using a virtual space such as a chat room might be a valuable

149 supplement to formal education during clinical education. To this end, Kakao Talk was

150 introduced as an informal educational tool in November 2014. On Monday mornings, the

151 hospital resident responsible for education created a chat room on Kakao Talk and invited

152 students and teachers to participate. At this time, students were informed that neither the extent

153 nor the content of their activities on Kakao Talk would influence their clinical scores/grades.

154 During the week, students could post questions in the chatroom. Teachers (either professors or

155 fellows) responded to each question as quickly as possible. The chat rooms were then closed on

156 Saturday morning of that week (Figure 1).

157 Research questions

158 Over the week-long clinical instruction period, we observed (without any quantification) that

unfamiliarity among students and between students and teachers on Kakao Talk during the early

part of a week induced fewer chat postings during that week. Therefore, we hypothesized that the 
161 sooner students began using Kakao Talk, the more they might use it overall and the more they 162 might use it for academic purposes (i.e., chat postings related to medicine, especially obstetrics

and gynecology, rather than social content). In this study, the relationship between the total number of student chats during the first two days of the week and the number of academic chats (defined as chats related to medicine) during the last three days was evaluated as the primary outcome. Moreover, the frequency with which students used Kakao Talk during the clinical education period and the factors associated with the frequency of this use were examined.

\section{Data collection and Analysis}

Thirty-nine weeks of online communication records of students' educational use of Kakao Talk were reviewed. A total of 39 clinical education groups $(n=151)$ were categorized into three sets (Low, $\mathrm{n}=13$; Middle, $\mathrm{n}=13$; High, $\mathrm{n}=13$ ) according to the number of total chats per student over five days. Next, the three categories (Category $1=$ Low, Category $2=$ Middle, Category $3=$ High) were determined according to the number of chats, sex ratio, season of clinical education (spring, summer, fall, winter), number of student questions, type of student questions (according to the subspecialty of obstetrics and gynecology), response time to student questions, and number of students (Table 1 and Table 2).

We used Poisson regression to estimate the scatter plot and correlation coefficient regarding the relationship between the number of total chats during the first and second days and the maximum value of academic chats during the third through fifth days. Categorical variables were expressed as counts and percentages and analyzed using Fisher's exact test. For data with normal distributions, continuous variables were expressed using the means \pm standard deviations, while medians and interquartile ranges were used for data with non-normal distributions. 
184 followed by Bonferroni's method for parametric data and using the Kruskal-Wallis test followed

185 by the two-tailed Mann-Whitney rank sum test for nonparametric data. The cut-off used to

determine statistical significance was $p<.05$. We used the open-source statistical software $\mathrm{R}$ version 3.3.0 (http://www.R-project.org) and SPSS version 23 (IBM Corporation, Inc., Chicago, IL, USA) for the computations.

\section{Results}

The characteristics of the 39 groups showed that there were more four-student groups, more groups with more males than females, and fewer winter groups than other types of groups.

Distribution according to the number of students and sex ratio of students in the clinical groups and according to season did not differ across the three categories (Table 1). Among participants in the third category (high number of chats), the following were all found to be significantly higher than in the other two categories: (1) the number of total and academic chats over all five days, the first two days, and the last three days, (2) the number of students posting total and academic chats, (3) the number of academic chats per student, (4) the number of student questions, and (5) the number of questions related to obstetrics, endocrinology, gynecology, and gynecological oncology (Table 2). The mean response time to student questions and the proportion of student questions to which teachers replied within 30 minutes to one hour of posting were not significantly different across categories $(p>.05$; data not shown (File S1)).

On the first day of the clinical education week, $82.1 \%$ of groups posted total chats, while on the second day, the percentage was $46.2 \%$, on the third day, $61.5 \%$, on the fourth day, $51.3 \%$, and on the fifth day, $41.0 \%$. The proportions of students posting total chats were $66.9 \%$ on the first day, $28.5 \%$ on the second day, $36.4 \%$ on the third day, $29.8 \%$ on the fourth day, and $25.2 \%$ 
207 on the fifth day. More total chats than academic chats were posted on the first and fifth days $(p=$ 208.001 and $p=.035$, respectively). On the second, third, and fourth days, the number of total chats 209 was similar to the number of academic chats $(p>.05$; data not shown (File S1)). The number of 210 total and academic chats, the number of students posting academic chats, and the number of total 211 chats per student posting total chats were not significantly different between the first two days 212 and the last three days. However, during the last three days, the number of students posting total 213 chats was lower than in the first two days, and the number of academic chats per student posting 214 academic chats was higher (Table 3). The number of total and academic chats per student was 215 not significantly different between the first two days and the last three days (total, median 216 [interquartile range]: $1.3[1.3]$ vs $1.3[2.1], p=.787$; academic: 0.3 [1.5] vs 1 [2], $p=.101)$. 217 Because of the extremely small number of total and academic chats per student, the number of 218 chats per student posting chats was evaluated.

To test whether the number of total chats during the first two days influenced the number of academic chats during the last three days, data on the groups in which the students posted chats during the first two days were analyzed. As shown in Figure 2A, the number of total chats posted on the first day significantly and positively correlated with the maximum value of academic chats on the third through fifth days (first day: correlation coefficient $=0.43, p=.02$ ). However, those posted on the second day (Figure 2B) showed a positive correlation with no statistical significance ( second day: correlation coefficient $=0.37, p=.17$ ). well as academic chats. Meanwhile, in the second category, all students in all groups except two posted total chats (median four students), while some students in all except one group (median two students) posted academic chats. By contrast, in the first category, some students in all 
230

231

232

233

234

235

236

237

238

239

240

241

242

243

244

245

246

247

248

249

250

251

252

except three groups posted total chats (median three students), and some students in all groups

except three (median one student) posted academic chats. We found that the number of total and academic chats was higher during the last three days than during the first two days in 11 groups in the third category (the number was equal in one group and lower in one group). This was also true of the second category in six groups (lower in seven groups), while in the first category, this was true for three groups (equal in one group and lower in nine groups). In all categories, the subject most frequently discussed in academic chats was the medical case allocated to each student, as well as procedures and delivery observed during clinical education and obstetrics and gynecology courses attended (data not shown (File S1)).

\section{Discussion}

In this exploratory pilot study, we retrospectively reviewed 39 weeks of online communication by 151 senior medical students related to their use of the Kakao Talk MIM service as an informal modality for education. The frequency with which the students used Kakao Talk during the first two days of a week of clinical education positively correlated with the frequency with which they used it for academic purposes during the last three days of that week. The number of students posting total chats decreased and the number of academic chats per student posting academic chats increased over one week of clinical education. In addition, the number of total chats per student positively correlated with the following: (1) the number of total and academic chats over all five days, the first two days, and the last three days, (2) number of students posting total and academic chats, (3) the number of academic chats per student, and (4) with the number and types of student questions.

Results from previous studies concerning the effectiveness of the use of MIM services 
253 in medical education vary. One prospective study on improving knowledge on breast cancer

254 among 25 hospital residents of gynecology and obstetrics reported that MIM services had a more

255 positive influence on learning and created more interest among learners than did booklets

256 (Alipour et al., 2012). In another prospective study, WhatsApp successfully facilitated a variety

257 of educational interactions in six groups of 19 third-year medical students (with one tutor per

258 group) during an eight-week clinical education period (Raiman, Antbring, \& Mahmood, 2017).

259 In a pilot study, WhatsApp was found to be useful in training 45 pathology residents (Goyal,

260 Tanveer, Sharma, 2017). However, in a randomized, multicenter small study, the use of

261 WhatsApp $(\mathrm{n}=32)$ was less effective for teaching medical residents than traditional e-learning

$262(\mathrm{n}=30)$, and it was suggested that its use served as a distraction (Clavier et al., 2019). In

263 addition, a recent study $(n=400)$ reported that while almost all medical students $(98 \%$ of the

$26497 \%$ who responded to the questionnaire) in the study used social media (WhatsApp, YouTube,

265 and Twitter), only a minority of students (40\%) used such platforms for academic purposes,

266 suggesting that the use of social media has no influence on academic performance (AlFaris et al.,

267 2018). In the current study, more than $25 \%$ of students continually used Kakao Talk during the

268 study period. During the middle of the clinical education week (second through fourth days),

269 students mainly used Kakao Talk for academic purposes. The number of students posting chats

270 and the number of chats per student who posted chats showed that Kakao Talk was more

271 frequently used for academic than for social purposes over a one-week period of clinical

272 education. Moreover, students who used Kakao Talk most often during the early period also used

273 it more often over time for academic purposes. These results demonstrate that using a MIM

274 service, such as Kakao Talk, might be useful in medical students' education but that the timing

275 of the start of this communication might also be a key factor in its effectiveness. 
277 Talk during clinical education was not influenced by teachers' response times to student questions. The students had different levels of activity on Kakao Talk, and many students used it

279 for academic purposes. The use of a MIM service by some, but not all, students might be due to their being unacquainted with one another and with their teachers on Kakao Talk during the early part of the clinical education period, which in turn, might influence their preference for MIM service use for academic purposes. Our results show a positive relationship between total activity on Kakao Talk during the early period and the number of academic chats during the later period, indicating that early chatting among students and teachers increases online chat activity overall followed by increased use for academic purposes.

The number of total and academic chats per student can be viewed as representing the extent of students' voluntary usage of Kakao Talk. Our data demonstrate that in the groups categorized into the third category, teamwork was stronger than in groups in other categories.

This is because all students participated in posting total chats, almost all students participated in posting academic chats, and the number of total and academic chats increased over time in most of these groups. Students in groups in the first category showed relatively weaker teamwork based on their low (individual) participation in posting chats (total and academic) and the decrease in the number of chats (total and academic) over time in many groups. Students with a stronger sense of teamwork might use Kakao Talk in an environment where they are encouraged to use this tool for social and academic purposes. This, in turn, might lead to relatively higher academic exposure for all students. In contrast, students with a weaker sense of teamwork might tend to use Kakao Talk individually in an environment where its use is not encouraged, which may lead to relatively lower academic exposure for all students. 
The limitations of our study include its retrospective nature and relatively short

300

301

302

303

304

305

306

307

308

309

310

311

312

313

314

observation period. Moreover, the efficacy of MIM services in student education was not directly

evaluated in relation to clinical performance, test scores, or other forms of assessment.

Nevertheless, by suggesting that the use of a popular MIM service might be related to an

eventual increase in its use for academic purposes within a very short period, this pilot study has potential as a basis for well-designed large-scale prospective trials.

\section{Conclusion}

This pilot study demonstrated that if MIM services are used frequently by medical students during the early part of a period of clinical education, they are more likely to be used for academic purposes later on. Our study also suggests that MIM services (particularly Kakao Talk) can be used for medical students' education, although it is an informal modality. Large-scale prospective trials are warranted to further evaluate the utility of MIM services.

\section{Acknowledgements}

Not applicable.

\section{References}

AlFaris E, Irfan F, Ponnamperuma G, Jamal A, Van der Vleuten C, Al Maflehi N, Al-Qeas S, Alenezi A, Alrowaished M, Alsalman R, Ahmed AMA. 2018. The pattern of social media use and its association with academic performance among medical students. Med Teach 
322

323

Sep;40(sup1):S77-S82

Alipour S, Moini A, Jafari-Adli S, Gharaie N, Mansouri K. 2012. Comparison of teaching about breast cancer via mobile or traditional learning methods in gynecology residents. Asian Pac J Cancer Prev 13:4593-5

Bouhnik D, Deshen M. 2014. WhatsApp goes to school: Mobile instant messaging between teachers and students. $J$ Inf Technol Edu Res 13:217-31

Clavier T, Ramen J, Dureuil B, Veber B, Hanouz JL, Dupont H, Lebuffe G, Besnier E, Compere V. 2019. Use of the Smartphone App WhatsApp as an E-Learning Method for Medical Residents: Multicenter Controlled Randomized Trial. JMIR Mhealth Uhealth Apr 9;7(4):e12825

Goyal A, Tanveer N, Sharma P. 2017. WhatsApp for Teaching Pathology Postgraduates: A Pilot Study. J Pathol Inform Feb 28;8:6

Han J, Cho O. 2015. Platform business Eco-model evolution: case study on KakaoTalk in Korea. J Open Innov 1:6 https://doi.org/10.1186/s40852-015-0006-8

Jamal A, Temsah MH, Khan SA, Al-Eyadhy A, Koppel C, Chiang MF. 2016. Mobile Phone Use Among Medical Residents: A Cross-Sectional Multicenter Survey in Saudi Arabia. JMIR Mhealth Uhealth May 19;4(2):e61

Lauricella S, Kay R. 2013. Exploring the use of text and instant messaging in higher education classrooms. Res Learn Technol 21:19061

Nay JR. 2013. KakaoTalk on 93 Percent of Smartphones in South Korea, Expecting \$200 Million in Revenue. Available at http://www.trutower.com (accessed 12 May 2019)

Raiman L, Antbring R, Mahmood A. 2017. WhatsApp messenger as a tool to supplement medical education for medical students on clinical attachment. BMC Med Educ 17:7 
345 So S. 2016. Mobile instant messaging support for teaching and learning in higher education.

$346 \quad$ Internet High Educ 31:32-42

347 Unuth N. 2019. KakaoTalk Free Calling and Messaging App Review. Available at 348 https://www.lifewire.com (accessed 12 May 2019)

349 Yeboah J, Ewur GD. 2014. The impact of WhatsApp messenger usage on students performance in 350 Tertiary Institutions in Ghana. J Educ Pract 5:157-64

351

352

353

354

355

356

357

358

359

360

361

362

Figure legends

363

Figure 1. Study design. Formal and informal education.

Figure 2. Scatter plots of maximum value of academic chats on the third through fifth days by the number of total chats on the first or second day. Scatter plots of A. First day and B. Second day. Frequency refers to the number of groups performing the same number of chats. 


\section{Table $\mathbf{1}$ (on next page)}

Table 1. Characteristics based on the number of total chats per student

$p$-value for the difference among the categories by multiple comparisons using the KruskalWallis test, followed by the 2-tailed Mann-Whitney rank sum test. Differences are reported between "category $1 \mathrm{v}$. category $2,{ }^{\dagger}$ category $2 \mathrm{v}$. category 3 , and ${ }^{\ddagger}$ category $1 \mathrm{v}$. category $3 .{ }^{\varsigma}$ $p$-value for differences among the categories using Fisher's exact test. 'Number of total chats per student' was defined as the total number of chats divided by the total number of students in each group. 
1 Table 1. Characteristics based on the number of total chats per student

\begin{tabular}{|c|c|c|c|c|c|}
\hline \multirow[b]{2}{*}{ Characteristic } & \multirow{2}{*}{$\begin{array}{c}\text { Total } \\
(n=39)\end{array}$} & \multicolumn{3}{|c|}{ Category } & \multirow[t]{2}{*}{$p$-value } \\
\hline & & $\begin{array}{c}1 \\
(n=13)\end{array}$ & $\begin{array}{c}2 \\
(n=13)\end{array}$ & $\begin{array}{c}3 \\
(n=13)\end{array}$ & \\
\hline \multicolumn{6}{|l|}{ Number of total chats per } \\
\hline $\begin{array}{l}\text { student, median (interquartile } \\
\text { range) }\end{array}$ & $2.3(2)$ & $1.3(0.6)$ & $2.3(0.7)$ & $6.5(5.4)$ & $0.000^{*}, \dagger, \ddagger$ \\
\hline Number of students, n (\%) & & & & & $0.416^{\S}$ \\
\hline Three & $8(20.5)$ & $1(2.5)$ & $4(10.3)$ & $3(7.7)$ & \\
\hline Four & $29(74.4)$ & $12(30.8)$ & $8(20.5)$ & $9(23.1)$ & \\
\hline Five & $2(5.1)$ & $0(0)$ & $1(2.5)$ & $1(2.5)$ & \\
\hline Sex ratio of students, $\mathrm{n}(\%)$ & & & & & $0.437 \S$ \\
\hline Equal (2:2) & $8(20.5)$ & $2(5.1)$ & $1(2.5)$ & $5(12.8)$ & \\
\hline Male $>$ female & $26(66.7)$ & $9(23.1)$ & $10(25.6)$ & $7(17.9)$ & \\
\hline Male $<$ female & $5(12.8)$ & $2(5.1)$ & $2(5.1)$ & $1(2.5)$ & \\
\hline Season, n (\%) & & & & & $0.278^{\S}$ \\
\hline
\end{tabular}




$\begin{array}{lcccc}\text { Spring } & 12(30.8) & 4(10.3) & 2(5.1) & 6(15.4) \\ \text { Summer } & 8(20.5) & 1(2.5) & 3(7.7) & 4(10.3) \\ \text { Fall } & 16(41.0) & 6(15.4) & 7(17.9) & 3(7.7) \\ & 3(7.7) & 2(5.1) & 1(2.5) & 0(0)\end{array}$

$2 \quad p$-value for the difference among the categories by multiple comparisons using the Kruskal-Wallis

3 test, followed by the 2-tailed Mann-Whitney rank sum test. Differences are reported between

4 *category $1 \mathrm{v}$. category 2 , †category $2 \mathrm{v}$. category 3 , and *ategory $1 \mathrm{v}$. category 3.

$5 \S p$-value for differences among the categories using Fisher's exact test.

6 'Number of total chats per student' was defined as the total number of chats divided by the total

7 number of students in each group. 


\section{Table 2 (on next page)}

Table 2. Student activity on Kakao Talk based on the number of total chats per student $p$-value for differences among the categories by multiple comparisons using one-way ANOVA, followed by the Bonferroni method. ${ }^{\varsigma} p$-value for the difference among the categories by multiple comparisons using the Kruskal-Wallis test, followed by the 2-tailed Mann-Whitney rank sum test. Differences are reported between ${ }^{*}$ category $1 \mathrm{v}$. category $2,{ }^{\dagger}$ category $2 \mathrm{v}$. category 3 , and ${ }^{\ddagger}$ category $1 \mathrm{v}$. category $3 .{ }^{\dagger}$ in total and academic student chats during first and second days were 0.071 and 0.081 , respectively. ${ }^{*}$ in total student chats during the last three days were 0.064. " Gynecology included urogynecology. 'Number of total chats per student' was defined as the total number of chats divided by the total number of students in each group. 'Number of academic chats per student' was defined as the number of academic chats divided by the total number of students in each group. 
1 Table 2. Student activity on Kakao Talk based on the number of total chats per student

\begin{tabular}{|c|c|c|c|c|c|}
\hline \multirow{3}{*}{ Student chat activity } & & \multicolumn{3}{|c|}{ Category } & \multirow[t]{3}{*}{$p$-value } \\
\hline & $(n=39)$ & 1 & 2 & 3 & \\
\hline & & $(n=13)$ & $(n=13)$ & $(n=13)$ & \\
\hline
\end{tabular}

Student chats

\begin{tabular}{|c|c|c|c|c|}
\hline Total, mean \pm SD & $12.7 \pm 10.8$ & $5.2 \pm 1.7$ & $8.9 \pm 2.0$ & $24 \pm 12.1$ \\
\hline Academic, mean $\pm \mathrm{SD}$ & $9.8 \pm 11.3$ & $3.5 \pm 3.2$ & $4.5 \pm 2.7$ & $21.5 \pm 12.9$ \\
\hline
\end{tabular}

Student chats during first and

second days

Total, mean \pm SD

Academic, median

(interquartile range)
$1(6)$

$0(3.5)$

$0(5)$

6(13.5)

Student chats during the last

three days, median

(interquartile range)

Total

Academic
$5(10)$

$3(8)$
$3.2 \pm 1.9$

$5.2 \pm 2.4$

$9.6 \pm 7.8$

$.006^{\dagger}$,

$.056^{\dagger+, \$ \S}$ 
Number of students posting

chats, median (interquartile

range)

Total

$4(1)$

$3(1.5)$

$4(1)$

$4(0)$

$.003^{*,+\infty}, \$$

Academic

$3(2)$

$1(2)$

$2(1)$

$4(0.5)$

$.000^{\dagger, t \&}$

Number of academic chats

per student, median

(interquartile range)

$1.4(2.2) \quad 0.5(1.1) \quad 1.0(1.2)$

$5.8(5.3)$

$.000 \dagger, \$$

Number of posted questions,

median (interquartile range)

$3(6)$

$1(2.5)$

$3(2)$

$9(11)$

$.000^{*}, \dagger$,

Types of posted questions,

median (interquartile range)

\begin{tabular}{|c|c|c|c|c|c|}
\hline Obstetrics & $1(2)$ & $0(1)$ & $1(1)$ & $3(4)$ & $.001^{\dagger+, \$}$ \\
\hline Endocrinology & $0(1)$ & $0(1)$ & $0(1)$ & $2(3)$ & $.000^{\dagger, t}$ \\
\hline Gynecology" & $0(1)$ & $0(1)$ & $1(2)$ & $2(3)$ & $.015^{*},+$, \\
\hline Gynecologic oncology & $1(2)$ & $0(1)$ & $0(1)$ & $3(3)$ & $.002^{\dagger, t, \S}$ \\
\hline
\end{tabular}


5 Wallis test, followed by the 2-tailed Mann-Whitney rank sum test.

6 Differences are reported between *category $1 \mathrm{v}$. category 2 , †category $2 \mathrm{v}$. category 3 , and

7 †ategory 1 v. category 3 .

$8 \dagger$ in total and academic student chats during first and second days were 0.071 and 0.081 ,

9 respectively.

$10 *$ in total student chats during the last three days were 0.064 .

11 Gynecology included urogynecology.

12 'Number of total chats per student' was defined as the total number of chats divided by the total

13 number of students in each group.

14 'Number of academic chats per student' was defined as the number of academic chats divided by 15 the total number of students in each group. 


\section{Table 3 (on next page)}

Table 3. Student activity on Kakao Talk during the first two days and the last three days of a clinical week

$p$-value for the difference between the first two days and the last three days using the 2tailed Mann-Whitney rank sum test. 'Number of total chats per student posting chats' was defined as the total number of chats divided by the total number of students posting chats in each group. 'Number of academic chats per student posting chats' was defined as the number of academic chats divided by the number of students posting academic chats in each group. 
1 Table 3. Student activity on Kakao Talk during the first two days and the last three days of

2 a clinical week

\begin{tabular}{lcccc}
\hline & Total & First two days & Last three days & \\
Student activity & $(n=39)$ & $(n=39)$ & $(n=39)$ & $p$-value \\
& & & \\
\hline
\end{tabular}

Student chats, median

(interquartile range)

Total

Academic

$6(8)$

$5(4)$

$5(10)$

.756

Number of students

posting chats, median

(interquartile range)

Total

$4(1)$

$3(2)$

$2(3)$

.038

Academic

$3(2)$

$1(3)$

$2(3)$

.243

Number of chats per

student posting chats,

median (interquartile

range)

Total

$3.5(1.25)$

$1.5(1)$

$2(1.75)$

.177 
Academic $2.5(2)$ $1(2)$

$3 \quad p$-value for the difference between the first two days and the last three days using the 2-tailed

4 Mann-Whitney rank sum test.

5 'Number of total chats per student posting chats' was defined as the total number of chats

6 divided by the total number of students posting chats in each group.

7 'Number of academic chats per student posting chats' was defined as the number of

8 academic chats divided by the number of students posting academic chats in each group. 
Figure 1

Figure 1. Study design. Formal and informal education.

Formal and informal education.

A.

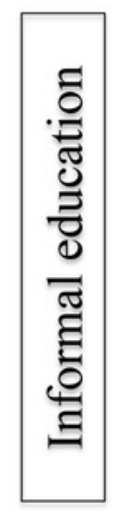

Questions and answers

\begin{tabular}{|c|}
\hline $\begin{array}{c}39 \text { Kakao-Talk } \\
\text { groups }\end{array}$ \\
\hline
\end{tabular}

- Monday morning: creation of a chat room

- Saturday morning: closure of a chat room

\section{Each group}

Students $(n=3-5)$

Professors $(n=2-3)$

Fellows $(\mathrm{n}=2)$

Resident $(\mathrm{n}=1)$

\section{Five-day course}

\section{9 students groups}
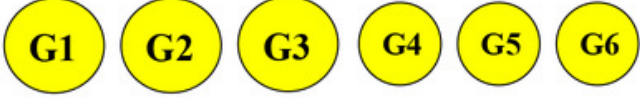

(G7)

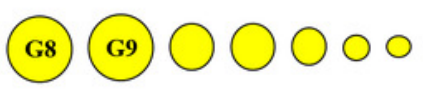

B.
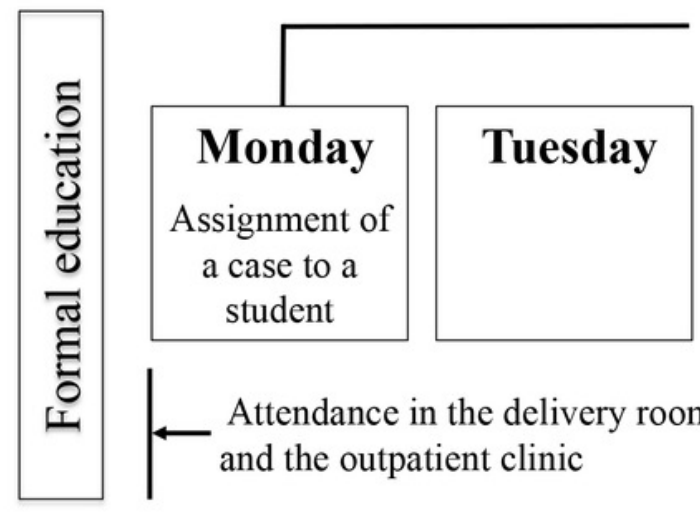

Five-day course
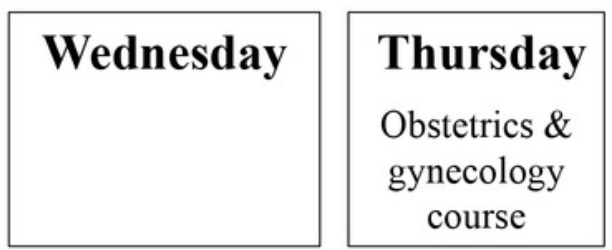

\section{Friday \\ Presentation about the assigned case}

Attendance in the delivery room, operating room, infertility intervention room, wards, and the outpatient clinic 
Figure 2

Figure 2. Scatter plots of maximum value of academic chats on the third through fifth days by the number of total chats on the first or second day.

Scatter plots of A. First day and B. Second day. Frequency refers to the number of groups performing the same number of chats. 

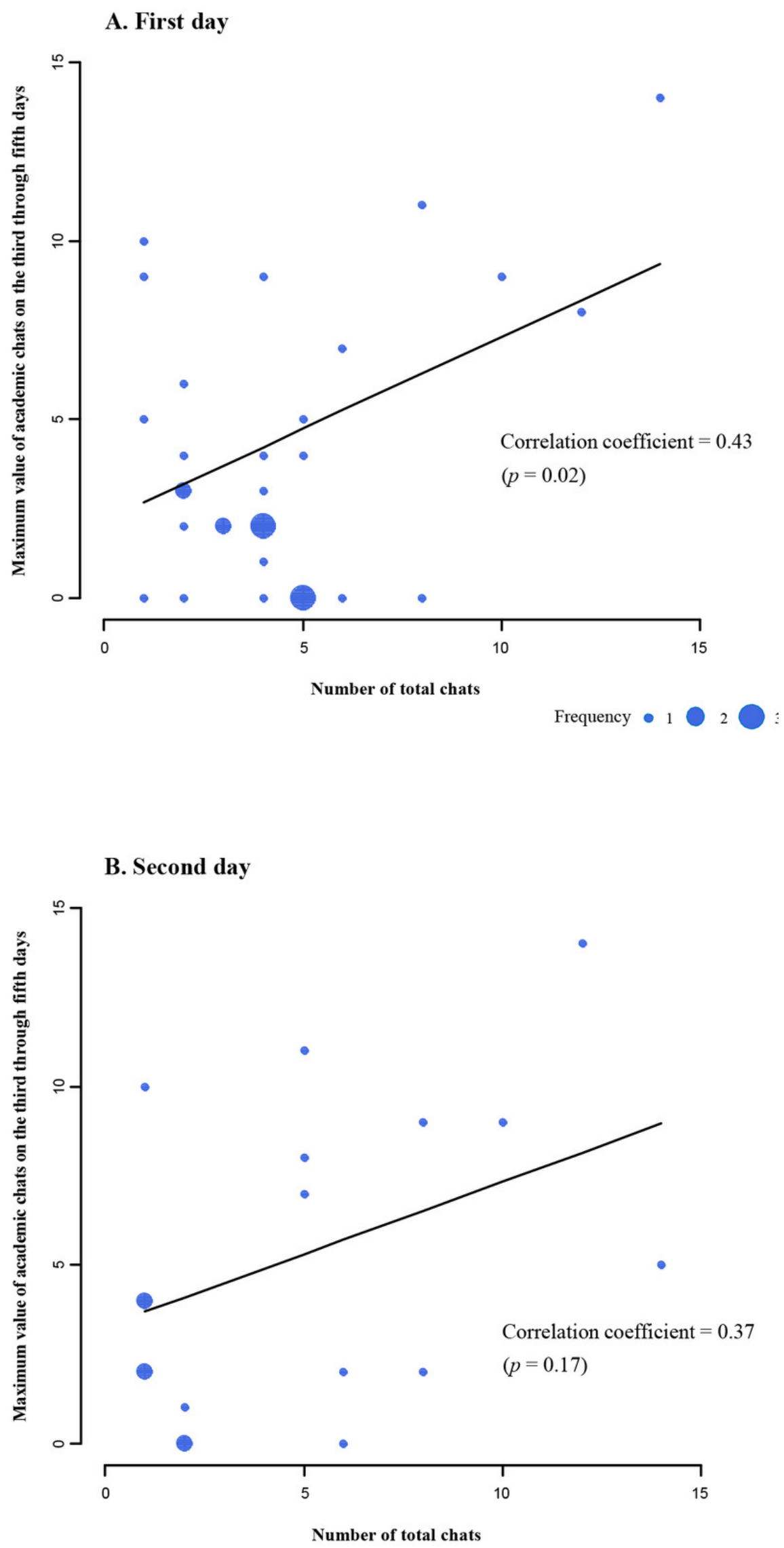

Frequency $\bullet 1 \bigcirc 2$

PeerJ reviewing PDF | (2019:01:34282:1:2:NEW 4 Jun 2019) 